\title{
Radon and material radiopurity assessment for the NEXT double beta decay experiment
}

\author{
S. Cebrián ${ }^{1,2}$, J. Pérez ${ }^{3}$, I. Bandac ${ }^{2}$, L. Labarga ${ }^{4}$, V. Álvarez ${ }^{5}$, A.I. Barrado ${ }^{6}$,
} A. Bettini ${ }^{2,7}$, F.I.G.M. Borges ${ }^{8}$, M. Camargo ${ }^{9}$, S. Cárcel ${ }^{5}$, A. Cervera ${ }^{5}$, C.A.N. Conde ${ }^{8}$, E. Conde ${ }^{6}$, T. Dafni ${ }^{1,2}$, J. Díaz ${ }^{5}$, R. Esteve ${ }^{10}$, L.M.P. Fernandes ${ }^{8}$, M. Fernández ${ }^{6}$, P. Ferrario ${ }^{5}$, E.D.C. Freitas ${ }^{11}$, L.M.P. Fernandes ${ }^{8}$, V.M. Gehman ${ }^{12}$, A. Goldschmidt ${ }^{12}$, J.J. Gómez-Cadenas ${ }^{5}$, D. González-Díaz ${ }^{1,2}$, R.M. Gutiérrez ${ }^{9}$, J. Hauptman ${ }^{13}$, J.A. Hernando Morata ${ }^{14}$, D.C. Herrera ${ }^{1,2}$, I.G. Irastorza ${ }^{1,2}$, A. Laing ${ }^{5}$, I. Liubarsky ${ }^{5}$, N. López-March ${ }^{5}$, D. Lorca ${ }^{5}$, M. Losada ${ }^{9}$, G. Luzón ${ }^{1,2}$, A. Marí1 ${ }^{10}$, J. Martín-Albo ${ }^{5}$, A. Martínez ${ }^{5}$, G. Martínez-Lema ${ }^{14}$, T. Miller ${ }^{12}$, F. Monrabal ${ }^{5}$, M. Monserrate ${ }^{5}$, C.M.B. Monteiro ${ }^{8}$, F.J. Mora ${ }^{10}$, L.M. Moutinho ${ }^{11}$, J. Muñoz Vidal ${ }^{5}$, M. Nebot-Guinot ${ }^{5}$, D. Nygren ${ }^{12}$, C.A.B. Oliveira ${ }^{12}$, A. Ortiz de Solórzano $^{1,2}$, J.L. Pérez Aparicio ${ }^{15}$, M. Querol ${ }^{5}$, J. Renner ${ }^{12}$, L. Ripoll ${ }^{16}$, J. Rodríguez ${ }^{5}$, F.P. Santos ${ }^{8}$, J.M.F. dos $\operatorname{Santos}^{8}$, L. Serra ${ }^{5}$, D. Shuman ${ }^{12}$, A. Simón ${ }^{5}$, C. Sofka ${ }^{17}$, M. Sorel ${ }^{5}$, J.F. Toledo ${ }^{10}$, J. Torrent ${ }^{16}$, Z. Tsamalaidze ${ }^{18}$, J.F.C.A. Veloso ${ }^{11}$, J.A. Villar ${ }^{1,2}$, R.C. Webb ${ }^{17}$, J.T. White ${ }^{17}$ and N. Yahlali ${ }^{5}$

${ }^{1}$ Laboratorio de Física Nuclear y Astropartículas, Universidad de Zaragoza, C/ Pedro Cerbuna 12, 50009 Zaragoza, Spain

${ }^{2}$ Laboratorio Subterráneo de Canfranc, Paseo de los Ayerbe s/n, 22880 Canfranc Estación, Huesca, Spain ${ }^{3}$ Instituto de Física Teórica, UAM/CSIC, Campus de Cantoblanco, 28049 Madrid, Spain

${ }^{4}$ Dpto. de Física Teórica, Universidad Autónoma de Madrid, Campus de Cantoblanco, 28049 Madrid, Spain

${ }^{5}$ Instituto de Física Corpuscular, CSIC \& Universitat de València, C/ Catedrático José Beltrán, 2, 46980 Paterna, Valencia, Spain

${ }^{6}$ Centro de Investigaciones Energéticas, Medioambientales y Tecnológicas, Complutense 40, 28040 Madrid, Spain

${ }^{7}$ Padua University and INFN Section, Dipartimento di Fisica G. Galilei, Via Marzolo 8, 35131 Padova, Italy

${ }^{8}$ Departamento de Fisica, Universidade de Coimbra, Rua Larga, 3004-516 Coimbra, Portugal

${ }^{9}$ Centro de Investigaciones en Ciencias Básicas y Aplicadas, Universidad Antonio Nariño, Carretera 3 este No. 47A-15, Bogotá, Colombia

${ }^{10}$ Instituto de Instrumentación para Imagen Molecular, Universitat Politècnica de València, Camino de Vera, $s / n$, Edificio 8B, 46022 Valencia, Spain

${ }^{11}$ Institute of Nanostructures, Nanomodelling and Nanofabrication, Universidade de Aveiro, Campus de Santiago, 3810-193 Aveiro, Portugal

${ }^{12}$ Lawrence Berkeley National Laboratory, 1 Cyclotron Road, Berkeley, California 94720, USA

${ }^{13}$ Department of Physics and Astronomy, Iowa State University, 12 Physics Hall, Ames, Iowa 50011-3160, USA

${ }^{14}$ Instituto Gallego de Física de Altas Energías, Univ. de Santiago de Compostela, Campus sur, Rúa Xosé María Suárez Núñez, s/n, 15782 Santiago de Compostela, Spain

${ }^{15}$ Dpto. de Mecánica de Medios Continuos y Teoría de Estructuras, Univ. Politècnica de València, Camino de Vera, s/n, 46071 Valencia, Spain

${ }^{16}$ Escola Politècnica Superior, Universitat de Girona, Av. Montilivi, s/n, 17071 Girona, Spain

${ }^{17}$ Department of Physics and Astronomy, Texas A\&M University, College Station, Texas 77843-4242, USA

${ }^{18}$ Joint Institute for Nuclear Research, Joliot-Curie 6, 141980 Dubna, Russia 


\begin{abstract}
The "Neutrino Experiment with a Xenon TPC" (NEXT), intended to investigate the neutrinoless double beta decay using a high-pressure xenon gas TPC filled with Xe enriched in ${ }^{136} \mathrm{Xe}$ at the Canfranc Underground Laboratory in Spain, requires ultra-low background conditions demanding an exhaustive control of material radiopurity and environmental radon levels. An extensive material screening process is underway for several years based mainly on gamma-ray spectroscopy using ultra-low background germanium detectors in Canfranc but also on mass spectrometry techniques like GDMS and ICPMS. Components from shielding, pressure vessel, electroluminescence and high voltage elements and energy and tracking readout planes have been analyzed, helping in the final design of the experiment and in the construction of the background model. The latest measurements carried out will be presented and the implication on NEXT of their results will be discussed. The commissioning of the NEW detector, as a first step towards NEXT, has started in Canfranc; in-situ measurements of airborne radon levels were taken there to optimize the system for radon mitigation and will be shown too.
\end{abstract}

Keywords: Double beta decay; Time-Projection Chamber (TPC); Gamma detectors (HPGe); Material radiopurity PACS: $14.60 . \mathrm{Pq}, 23.40 .-\mathrm{s}, 29.40 .-\mathrm{n}$

\title{
INTRODUCTION
}

The observation of neutrinoless double beta decay would be outstanding for characterizing neutrino properties [1]. The NEXT experiment ("Neutrino Experiment with a Xenon Time-Projection Chamber") aims to search for such a decay in ${ }^{136} \mathrm{Xe}$ at the Laboratorio Subterráneo de Canfranc (LSC), located at the Spanish Pyrenees, with a source mass of $\sim 100 \mathrm{~kg}$ (NEXT-100 phase). The challenge is to combine, while keeping the detector=source approach, the measurement of the topological signature of the event (in order to discriminate the signal from background) with the energy resolution optimization (to single out the peak at the sum energy of the two emitted electrons). The NEXT detector will be a high pressure gaseous xenon Time-Projection Chamber (TPC) with proportional electroluminescent (EL) amplification [2]. There will be separate energy and tracking readout planes, located at opposite sides of the pressure vessel, using different sensors: photomultiplier tubes (PMTs) for calorimetry (and for fixing the start of the event) and silicon photomultipliers (SiPMs) for tracking. While work on prototypes is still ongoing [3], the installation of shielding and ancillary system started at LSC in 2013. Underground commissioning of the NEW detector began at the end of 2014 and first data are expected along 2015. The NEW (NEXT-WHITE) apparatus ${ }^{1}$ is the first phase of the NEXT detector to operate underground; it is a downscale 1:2 in size (1:8 in mass) of NEXT-100.

The goal of NEXT is to explore electron neutrino effective Majorana masses below $100 \mathrm{meV}$ for a total exposure of $500 \mathrm{~kg}$.year. To reach this sensitivity, there are two basic requirements: 1) An energy resolution of at most 1\% FWHM at the transition energy $\left(\mathrm{Q}_{\beta \beta}=2.458 \mathrm{MeV}\right)$, which is reachable with EL amplification according to the results of prototypes. 2) A background level below $8 \times 10^{-4}$ counts $\mathrm{keV}^{-1} \mathrm{~kg}^{-1} \mathrm{y}^{-1}$ in the energy region of interest, achievable thanks to passive shieldings, pattern recognition techniques and a thorough material radiopurity control. Materials to be used in the whole experimental set-up have been screened and first results presented in $[4,5,6,7,8]$. Here, new results are shown and discussed together with the first direct quantification of radon levels at the NEXT site in Canfranc.

\section{MATERIAL RADIOASSAY}

The material screening program of the NEXT experiment is mainly based on germanium $\gamma$-ray spectrometry using ultra-low background detectors operated deep underground, at a depth of 2450 m.w.e., from the Radiopurity Service of LSC; being a non-destructive technique, the actual components to be used in the experiment can be analyzed. Detectors are p-type close-end coaxial 2.2-kg High Purity germanium detectors, from Canberra France. For the measurements presented here, the shielding consisted of 5 or $10 \mathrm{~cm}$ of copper in the inner part surrounded by $20 \mathrm{~cm}$ of low activity lead, with boil-off nitrogen flush to avoid airborne radon intrusion. Detection efficiency is estimated for each sample by GEANT4 simulation. To complement germanium spectrometry results, measurements based on Glow Discharge Mass Spectrometry (GDMS) and Inductively Coupled Plasma Mass Spectrometry (ICPMS) have been also carried out. GDMS is performed by Evans Analytical Group in France, providing concentrations of U, Th and K. ICPMS measurements were made at CIEMAT (Unidad de Espectrometria de Masas) in Spain.

\footnotetext{
${ }^{1}$ The name honours the memory of the late Professor James White, key scientist of the NEXT project.
} 
Materials analyzed deal with the shielding, pressure vessel, field cage and EL components and the energy and tracking readout planes. Results obtained after those already presented in [4]-[8] are summarized in table 1 and described in the following; for germanium measurements, reported errors correspond to $1 \sigma$ uncertainties and upper limits are given at $95 \%$ C.L.. Uncertainties for GDMS results are typically of $20 \%$.

The passive shielding of NEXT consists of a 20-cm-thick lead castle together with an additional 12-cm-thick copper layer placed inside the vessel. Lead and copper from different suppliers were studied [4, 5, 7]. The selected options were CuA1 (or ETP) copper from Lugand Aciers and refurbished lead from the OPERA experiment, following the analysis by GDMS and using Ge detectors [7]. After the melting of the OPERA lead sheets for brick production by Tecnibusa company a new screening of the material was performed (row \#1 of table 1); no contamination was introduced in the process and the nominal value of $80 \mathrm{~Bq} / \mathrm{kg}$ for ${ }^{210} \mathrm{~Pb}$ activity [9] was verified by measuring $(76.4 \pm 8.6) \mathrm{Bq} / \mathrm{kg}$ using the $46.5 \mathrm{keV}$ line. Different types of steel used in the lead castle structure have been analyzed. After screening samples of the 316Ti stainless steel from Nironit used for walls [5], structural steel S-275 was also measured; three samples were taken into consideration, made of the raw steel, steel after coating with antioxidant primer and after additional painting (rows \#2-4 of table 1). The primer seems to be responsible for the high activities observed. Materials intended to be used for filling empty space in the shielding were also screened: lead wool from Tecnibusa and foam joints made of EPDM sponge from Moss Express and Artein Gaskets (rows \#5-7 of table 1). It has been checked that the activity measured for these components should not have an impact on the background model of the experiment.

For the pressure vessel of NEXT-100, which must hold $15 \mathrm{~b}$, several samples of $316 \mathrm{Ti}$ stainless steel from Nironit were initially screened with germanium detectors [4] for body, end-caps and flanges and complementary results were obtained afterwards from GDMS analysis [7]. The TIG-MIG welding was also analyzed [5] and recently a sample of glue intended to be used on the tests of the vessel welding was screened (row \#8 of table 1).

The field cage is made using copper rings connected to resistors and High Density polyethylene as insulator; wire meshes separate the different field regions and reflector panels made of PTFE and coated with a wavelength shifter (TPB) improve the light collection. Many types of plastics (peek, semitron, kynar, tefzel and poliethylene from different suppliers) were screened and HD polyethylene was analyzed also by ICPMS [4, 5, 7]. ETP copper for field cage, in rod and sheet, was measured using GDMS [7] and now results for HV chip resistors from Ohmcraft can be presented too (row \#9 of table 1); activities higher than expected were found and more radiopure resistors will be searched for NEXT-100.

The energy readout plane of NEXT-100 will include 60 Hamamatsu R11410-10 PMTs placed behind the cathode to detect EL light and primary scintillation. Each PMT is sealed into individual, pressure resistant copper cans, coupled to the sensitive volume through a sapphire window coated with TPB. Shappire windows, copper for cans and plates and several components for PMT bases (capacitors, resistors, pin receptacles and thermal epoxy) were analyzed [5, 7]. Other items have been taken into consideration using Ge detectors or by GDMS analysis and results are presented in table 1: brazing paste (made of $72 \% \mathrm{Ag}$ and $28 \% \mathrm{Cu}$, row \#10), M4 stainless steel vented screws and brass bolts (for PMT cans, rows \#11-12), the optical coupling gel (SmartGel NyoGel OCK-451, row \#13), other epoxy (Araldite 2011, mixing resin and hardener, row \#14) and kapton-copper cable (used at bases, row \#15). U and Th concentration of brass bolts are about two orders of magnitude lower than those of stainless steel screws and therefore brass units have been selected. For Araldite 2011 epoxy, the obtained results are more stringent than those available at [10]. Presence of ${ }^{108 m} \mathrm{Ag}$ was also identified in the kapton-copper cable. The available PMTs of the selected model for NEXT-100 are being screened in 3-unit groups using the same Ge detector; since all of them show equivalent activity, a joint analysis of fifteen available runs (corresponding to 45 PMTs) has been carried out to derive activities per PMT (row \#16 of table 1). The results are compatible with those presented by XENON [11] and PandaX collaborations [12]; the same PMT model has been also analyzed in [13, 14]. The modified version R11410-21 of the Hamamatsu PMTs presents a ${ }^{60}$ Co activity reduced by a factor $\sim 4-5$, according to XENON1T results [15].

The tracking readout plane of NEXT-100 will consist of an array of 110 boards (named "Dice Boards", DB) placed behind the EL region. Each DB contains $8 \times 8 \mathrm{SiPM}$ sensors with a pitch of $\sim 1 \mathrm{~cm}$ and is coated with TPB. The design of a radiopure tracking plane, in direct contact with the gas detector medium, was a challenge since the needed components have typically activities too large for experiments requiring ultra-low background conditions; results of the radiopurity assessment for the tracking plane are presented in [8]. The substrate of the DBs is made of kapton and copper from Flexible Circuits Inc., showing better radiopurity than cuflon boards. SiPMs from SensL company (MLP, Moulded Lead-frame Package plastic SMT elements, MicroFC-10035-SMT-GP) were chosen after successful radioassay. Different electronic components (capacitors, connectors, solder paste, NTC temperature sensors and blue LEDs) were also screened with germanium detectors. New results have been obtained for the board-to-board connectors (HIROSE FX11LA-140S-SV, receptacles and HIROSE FX11LA-140P-SV, headers, row \#17 of table 1) 
finding similar values to those obtained for other connectors also made of LCP [8]; these connectors will be placed behind a copper shield. In an attempt to identify the origin of the measured activity in the kapton-Cu DBs (even if acceptable according to the background model of NEXT-100) the adhesive films used in these DBs were separately screened (row \#18 of table 1); the observed emissions cannot explain the activity of the boards.

\section{CONTROL OF RADON}

A series of measurements of radon activity levels and gamma background counting rates at the NEXT site in Canfranc has been performed to quantify and understand the evolution of these background components, with the aim to efficiently design their mitigation, profiting from the fact of having the external lead shielding of NEXT assembled at LSC without the detector mounted inside for several months. For first radon measurements an AlphaGuard counter from LSC was used inside NEXT lead castle, together with another one which takes data routinely at hall A, allowing to monitor radon evolution. To quantify the gamma background a simple set-up was prepared, consisting of a $3^{\prime \prime} \times 3^{\prime \prime}$ low background $\mathrm{NaI}(\mathrm{Tl})$ detector followed by a Canberra 2005 preamplifier, a Tennelec linear amplifier and a Canberra 8701 ADC, read by an Arduino board. In this way, energy of events up to $\sim 3 \mathrm{MeV}$ was continuously registered.

From October 2014 to February 2015, data were taken simultaneously by radon and gamma detectors at the following distinct conditions:

1. With the lead castle open.

2. After closing the lead castle.

3. After improving the conditions for closing the lead castle by blocking holes and using additional foam joints.

4. After performing a $\mathrm{N}_{2}$ purge, injecting a high flux of $\mathrm{N}_{2}$ gas $(1800 \mathrm{l} / \mathrm{h})$ for about seven hours. In this way, the total $\mathrm{N}_{2}$ gas injected was twice the internal volume of the shielding.

5. With the injection of a low constant $\mathrm{N}_{2}$ gas flux $(180 \mathrm{l} / \mathrm{h})$, set after performing a second $\mathrm{N}_{2}$ purge equivalent to the first one.

6. After stopping the constant $\mathrm{N}_{2}$ gas flux in situation 5 .

7. With the injection of a high constant $\mathrm{N}_{2}$ gas flux $(900 \mathrm{l} / \mathrm{h})$, set also after a new $\mathrm{N}_{2}$ purge equivalent to the previous ones.

Table 2 shows the total counting rate of the $\mathrm{NaI}(\mathrm{Tl})$ detector obtained at all the conditions of measurement, during stable periods following the different actions performed. Radon mean activity values, measured by the AlphaGuard detectors at these conditions both at hall A and inside the lead NEXT castle, are given in table 2 too; the corresponding standard deviations are indicated. By closing the castle, the gamma rate was reduced two orders of magnitude and the radon activity more than a factor 2 , becoming insensitive to external fluctuations. $\mathrm{A} \mathrm{N}_{2}$ purge and even a low constant $\mathrm{N}_{2}$ gas flux produced only marginal reductions. A high constant $\mathrm{N}_{2}$ gas flux allowed a more significant reduction, of at least one order of magnitude for the radon activity inside the castle; indeed, the sensitivity limit of the AlphaGuard detector was reached in that situation. A more sensitive radon detector would be necessary to quantify the actual reduction thanks to this high constant $\mathrm{N}_{2}$ gas rate; data were taken contemporaneously also using a custom-made radon detector with micromegas readout and analysis of these data is underway.

\section{SUMMARY}

An extensive screening program for materials and components used in the NEXT experiment is underway since 2011, based mainly on ultra-low background gamma spectrometry using HPGe detectors at LSC but also on complementary results from GDMS and ICPMS. Information on radiopurity obtained has helped in the design of the set-up: adequate materials for shieldings, vessel and field cage have been identified and a thorough selection of in-vessel components for energy and tracking planes has been performed too, checking radiopurity of all photomultiplier units and choosing finally kapton-copper boards and optimum SiPMs. Measured activity of the relevant radioisotopes has been used also as input in the construction of the background model of NEXT-100 [16]; according to first results, the required background level seems to be at reach.

On the other hand, airborne radon activity and gamma background rates have been measured using different detectors at various conditions inside the NEXT lead castle in Canfranc, before the commissioning of the NEW detector there, in order to optimize the radon suppression system. 
TABLE 1. Activities recently measured in relevant materials for NEXT. GDMS results were derived from U, Th and K concentrations. Germanium $\gamma$-ray spectrometry results reported for ${ }^{238} \mathrm{U}$ and ${ }^{232} \mathrm{Th}$ correspond to the upper part of the chains (derived from ${ }^{234 m} \mathrm{~Pa}$ and ${ }^{228} \mathrm{Ac}$ emissions) and those of ${ }^{226} \mathrm{Ra}$ and ${ }^{228} \mathrm{Th}$ give activities of the lower parts.

\begin{tabular}{|c|c|c|c|c|c|c|c|c|c|c|c|c|}
\hline & Material & Supplier & Technique & units & ${ }^{238} \mathrm{U}$ & ${ }^{226} \mathrm{Ra}$ & ${ }^{232} \mathrm{Th}$ & ${ }^{228} \mathrm{Th}$ & ${ }^{235} \mathrm{U}$ & ${ }^{40} \mathrm{~K}$ & ${ }^{60} \mathrm{Co}$ & ${ }^{137} \mathrm{Cs}$ \\
\hline 1 & Lead & Britannia & $\mathrm{Ge}$ & $\mathrm{mBq} / \mathrm{kg}$ & $<126$ & $<2.8$ & & $<3.2$ & & $<6.9$ & $<0.3$ & \\
\hline 2 & S-275 steel & Proycon & $\mathrm{Ge}$ & $\mathrm{mBq} / \mathrm{kg}$ & $32 \pm 9$ & $1.2 \pm 0.1$ & $1.9 \pm 0.2$ & $4.7 \pm 0.3$ & & $3.2 \pm 0.7$ & $1.8 \pm 0.1$ & $<0.2$ \\
\hline 3 & Steel+primer & Proycon & $\mathrm{Ge}$ & $\mathrm{mBq} / \mathrm{kg}$ & $(1.1 \pm 0.3) \times 10^{3}$ & $444 \pm 21$ & $125 \pm 9$ & $106 \pm 6$ & & $(1.6 \pm 0.2) \times 10^{3}$ & $94 \pm 7$ & $<3.9$ \\
\hline 4 & $\begin{array}{l}\text { Steel+primer } \\
\text { +painting }\end{array}$ & Proycon & $\mathrm{Ge}$ & $\mathrm{mBq} / \mathrm{kg}$ & $(0.8 \pm 0.2) \times 10^{3}$ & $437 \pm 20$ & $76 \pm 5$ & $58 \pm 3$ & & $(1.2 \pm 0.1) \times 10^{3}$ & $2.2 \pm 0.3$ & $<1.4$ \\
\hline 5 & Lead wool & Tecnibusa & $\mathrm{Ge}$ & $\mathrm{mBq} / \mathrm{kg}$ & $<368$ & $<12$ & & $<15$ & & $36.9 \pm 6.5$ & $<1.1$ & \\
\hline 6 & EPDM foam & Moss Express & $\mathrm{Ge}$ & $\mathrm{mBq} / \mathrm{m}$ & $<437$ & $33.0 \pm 1.7$ & $106 \pm 7$ & $95.5 \pm 5.2$ & & $758 \pm 78$ & $<1.4$ & $<1.3$ \\
\hline 7 & EPDM foam & Artein Gaskets & $\mathrm{Ge}$ & $\mathrm{mBq} / \mathrm{m}$ & $<215$ & $4.3 \pm 0.4$ & $5.1 \pm 1.6$ & $<5.4$ & & $11.2 \pm 2.9$ & $<0.6$ & $<0.6$ \\
\hline 8 & Glue & Ceys & $\mathrm{Ge}$ & $\mathrm{mBq} / \mathrm{kg}$ & $<3.2 \times 10^{3}$ & $<18$ & $<75$ & $<31$ & $<13$ & $(33.0 \pm 3.3) \times 10^{3}$ & $<12$ & $<10$ \\
\hline 9 & Resistors & Ohmcraft & $\mathrm{Ge}$ & $\mu \mathrm{Bq} / \mathrm{pc}$ & $\begin{array}{l}(0.56 \\
\pm 0.15) \times 10^{3} \\
\end{array}$ & $217 \pm 10$ & $44 \pm 4$ & $36 \pm 3$ & & $95 \pm 13$ & $<2$ & $<2$ \\
\hline 10 & Brazing paste & & GDMS & $\mu \mathrm{Bq} / \mathrm{kg}$ & $55 \pm 10$ & & $49 \pm 4$ & & & $<31$ & & \\
\hline 11 & Brass bolts & & GDMS & $\mu \mathrm{Bq} / \mathrm{kg}$ & $8.9 \pm 0.7$ & & $6.9 \pm 0.2$ & & & $<31$ & & \\
\hline 12 & SS screws & & GDMS & $\mathrm{mBq} / \mathrm{kg}$ & $3.25 \pm 0.25$ & & $0.57 \pm 0.08$ & & & $<0.19$ & & \\
\hline 13 & Optical gel & Nye Lubricants & $\mathrm{Ge}$ & $\mathrm{mBq} / \mathrm{kg}$ & $<1.7 \times 10^{3}$ & $<22$ & $<49$ & $<18$ & $<16$ & $<173$ & $<4.5$ & $<5.8$ \\
\hline 14 & Epoxy & Araldite & $\mathrm{Ge}$ & $\mathrm{mBq} / \mathrm{kg}$ & $<182$ & $<1.4$ & $<3.7$ & $<2.5$ & $<0.8$ & $15.0 \pm 2.4$ & $<0.4$ & $<0.4$ \\
\hline 15 & Kapton- $\mathrm{Cu}$ cable & & $\mathrm{Ge}$ & $\mathrm{mBq} / \mathrm{kg}$ & $<1.1 \times 10^{3}$ & $46.8 \pm 3.3$ & $<40$ & $<32$ & & $166 \pm 27$ & $<5.2$ & $<4.4$ \\
\hline 16 & $\begin{array}{l}\text { PMTs } \\
\text { (R11410-10) }\end{array}$ & Hamamatsu & $\mathrm{Ge}$ & $\mathrm{mBq} / \mathrm{pc}$ & $<67$ & $<0.94$ & $<2.2$ & $0.56 \pm 0.14$ & $0.58 \pm 0.13$ & $11.8 \pm 1.7$ & $3.73 \pm 0.27$ & $<0.3$ \\
\hline 17 & Connectors & Hirose & $\mathrm{Ge}$ & $\mathrm{mBq} / \mathrm{pc}$ & $6.4 \pm 1.9$ & $2.8 \pm 0.1$ & $5.6 \pm 0.3$ & $5.9 \pm 0.3$ & & $3.4 \pm 0.4$ & $<0.03$ & $<0.04$ \\
\hline 18 & Adhesive films & Flexible Circuits & $\mathrm{Ge}$ & $\mathrm{mBq} / \mathrm{kg}$ & $(1.8 \pm 0.6) \times 10^{3}$ & $<19$ & $<50$ & $<34$ & $16.8 \pm 3.0$ & $<107$ & $<4.8$ & $<4.6$ \\
\hline
\end{tabular}


TABLE 2. Summary of measurements taken at different conditions at the NEXT site in Canfranc: total rate registered by the $\mathrm{NaI}(\mathrm{Tl})$ detector and radon mean activities measured by the AlphaGuard detectors both at hall $\mathrm{A}$ and inside the lead castle. Standard deviations $(\sigma)$ for radon activity are also shown.

\begin{tabular}{|c|c|c|c|c|c|c|}
\hline & Situation & $\begin{array}{c}\mathrm{NaI}(\mathrm{Tl}) \text { detector rate } \\
(\mathrm{Hz})\end{array}$ & $\begin{array}{c}\text { Rn activity } \\
\left(\mathrm{Bq} / \mathrm{m}^{3}\right) \\
\text { mean }\end{array}$ & $\begin{array}{c}\text { at Hall A } \\
\sigma\end{array}$ & $\begin{array}{c}\text { Rn activity } \\
\left(\mathrm{Bq} / \mathrm{m}^{3}\right) \\
\text { mean }\end{array}$ & $\begin{array}{c}\text { at NEXT castle } \\
\sigma\end{array}$ \\
\hline 1 & Open castle & $59.56 \pm 0.01$ & & & & \\
\hline 2 & Closed castle & $1.089 \pm 0.001$ & 80 & 29 & 79 & 28 \\
\hline 3 & Better closed castle & $0.694 \pm 0.002$ & 74 & 26 & 30 & 11 \\
\hline 4 & After $\mathrm{N}_{2}$ purge & $0.658 \pm 0.001$ & 66 & 25 & 30 & 12 \\
\hline 5 & $\mathrm{~N}_{2}$ purge+flux $180 \mathrm{l} / \mathrm{h}$ & $0.600 \pm 0.001$ & 66 & 25 & 22 & 9 \\
\hline 6 & Without $\mathrm{N}_{2}$ flux & $0.638 \pm 0.001$ & 59 & 18 & 26 & 10 \\
\hline 7 & $\mathrm{~N}_{2}$ purge+flux $900 \mathrm{l} / \mathrm{h}$ & $0.350 \pm 0.002$ & 73 & 26 & 4 & 3 \\
\hline
\end{tabular}

\section{ACKNOWLEDGMENTS}

The NEXT Collaboration acknowledges funding support from the following agencies and institutions: the European Research Council under the Advanced Grant 339787-NEXT and the T-REX Starting Grant ref. ERC-2009-StG-240054 of the IDEAS program of the 7th EU Framework Program; the Spanish Ministerio de Economía y Competitividad under grants CONSOLIDER-Ingenio 2010 CSD2008-0037 (CUP), FPA2009-13697-C04-04, and FIS2012-37947C04; the Director, Office of Science, Office of Basic Energy Sciences of the US DoE under Contract no. DE-AC0205CH11231; and the Portuguese FCT and FEDER through the program COMPETE, Projects PTDC/FIS/103860/2008 and PTDC/FIS/112272/2009. S. Cebrián acknowledges the support from the University of Zaragoza (Convocatoria propia de proyectos de investigación para Jóvenes Investigadores 2014). Special thanks are due to LSC directorate and staff for their strong support for performing the measurements at the LSC Radiopurity Service.

\section{REFERENCES}

1. J.J. Gomez-Cadenas et al, Riv. Nuovo Cim. 35, 29-98 (2012).

S. R. Elliott, Mod. Phys. Lett. A 27, 1230009 (2012).

F. T. Avignone III et al., Rev. Mod. Phys. 80, 481 (2008).

O. Cremonesi and M. Pavan, Advances in High Energy Physics 2014, 951432 (2014).

2. J. J. Gomez Cadenas et al, Advances in High Energy Physics 2014, 907067 (2014).

3. V. Alvarez et al., Nucl. Instrum. Meth. A 708, 101-114 (2013).

V. Alvarez et al., JINST 8, P04002 (2013).

V. Alvarez et al., JINST 9, P03010 (2014).

4. V. Alvarez et al, JINST 8, T01002 (2013).

5. V. Alvarez et al, AIP Conf. Proc. 1549, 46 (2013) .

6. J. Pérez et al., Proceedings of Science (EPS-HEP 2013), 528 (2013).

7. T. Dafni et al, to appear in Nucl. Phys. B (PS), arXiv:1411.1222 [physics.ins-det].

8. V. Alvarez et al, JINST, in press (2015), arXiv:1411.1433 [physics.ins-det].

9. A. Anokhina et al, JINST 3, P07002 (2008).

10. J. Busto et al, Nucl. Instrum. and Meth. A 492, 35-42 (2002).

11. E. Aprile et al, Astropart. Phys. 35, 43 (2011).

L. Baudis et al, JINST 8 P04026 (2013).

12. X. G. Cao et al, arXiv:1405.2882v1 [physics.ins-det].

13. D. S. Akerib et al, Nucl. Instrum. and Meth. A 703, 1-6 (2013).

14. K. Lung et al, Nucl. Instrum. and Meth. A 696, 32-39 (2012).

15. E. Aprile et al, arXiv:1503.07698v1 [astro-ph.IM].

16. M. Nebot-Guinot, for the NEXT collaboration, to appear in Nucl. Phys. B (PS), arxiv:1410.6699. 